\title{
Sensitivity to Molar Feedback Functions: A Test of Molar Optimality Theory
}

\author{
R. H. Ettinger and Alliston K. Reid \\ Eastern Oregon State College
}

\author{
J. E. R. Staddon \\ Duke University
}

\begin{abstract}
Molar optimality models assume that any reward schedule can be described by a molar feedback function, which is the relation between average response rates and average reinforcement rates enforced by that particular schedule. This molar feedback function is considered, by optimality models, to be a sufficient description of the schedule for the prediction of steady-state molar performance. In this article we challenge the fundamental assumption of all molar optimality modelsthat animals are directly sensitive to this molar feedback function. We found that animals were sensitive to the schedule conditions in effect, especially at the molecular level of postfood time, but they were not directly sensitive to the slopes of any of the molar feedback functions that we manipulated. Our data do not simply represent a failure to maximize a particular utility function so that this form of the function requires alteration. Rather, they demonstrate that animals may not be sensitive to the molar rates of responding and reinforcement described by the molar feedback functions. Our animals were sensitive to the schedules at a molecular level, and it is to this molecular level that we should direct our attention.
\end{abstract}

Operant behavior can be explained in terms of its antecedents (causal accounts) or its consequences (functional accounts). The best developed functional accounts are in terms of optimality theory. Consequences can be measured moment by moment (molecular accounts) or as averages (molar accounts). Molar optimality accounts measure consequences in terms of the molar feedback function (MFF: Baum, 1973; Kagel, Battalio, Green, \& Rachlin, 1980; Rachlin \& Burkhard, 1978; Staddon, 1979; Timberlake, 1980), that is, the relation between average response rates and average reinforcement rates enforced by a particular schedule. It is possible to derive MFFs for all common reinforcement schedules. For example, the MFF for ratio schedules is a straight line through the origin, because reinforcement rate is directly proportional to response rate. The MFF for variable-interval schedules is a positively accelerated function with an asymptote at the scheduled maximum reinforcement rate, because reinforcement rate is directly related to response rate, but with diminishing returns.

Molar optimality theories relate the differences in molar performance engendered by different schedules to the properties of the MFFs associated with them. For example, Staddon's (1979) minimum-distance model and Rachlin and Burkhard's (1978) value-maximizing model both incorporate the MFF as a constraint to the objective function to be maximized or minimized. Any success of optimality theories in explaining performance

These experiments were supported in part by a grant from the National Science Foundation to J. E. R. Staddon.

The authors would like to thank Tracey Knock and Kevin McCadden for patiently helping with the animals, and Will Vaughan, William Baum, Howard Rachlin, and Dan Lloyd for comments on earlier drafts.

Correspondence concerning this article should be addressed to $\mathrm{R}$. $\mathrm{H}$. Ettinger or Alliston K. Reid at the Department of Psychology, Eastern Oregon State College, La Grande, Oregon 97850, or to J. E. R. Staddon at the Department of Psychology, Duke University, Durham, North Carolina 27706 differences between different schedules implies that animals are in some way sensitive to MFF properties such as slope (cf. Staddon, 1982). As the slope of the MFF is changed, the slope of the corresponding response function should also change. Nevertheless, several researchers have argued against the idea that animals can directly assess MFF slope--either on quantitative grounds (Prelec, 1982) or on the grounds of memory constraints (Hinson \& Staddon, 1983). No one, however, has directly measured the effects of changes in MFF slope on the slopes of the corresponding response functions in a way that tests sensitivity to the MFF slope. It, therefore, seemed worthwhile to manipulate MFF slope directly and observe the resulting changes in molar performance.

In Experiment 1 we looked at the effects on behavior of variation in the slope and the intercept of a set of positive, linear MFFs; in Experiment 2 we looked at the effects of variation in the slope and the intercept of a set of negative, linear MFFs.

\section{Experiment 1}

Response functions define the steady-state relation between obtained food and response rates over a set of molar feedback functions that vary in some systematic way (a set of different variable-interval schedules, for example). In our first experiment we examined the form of response functions generated by three sets of linear MFFs with positive slopes. These MFFs were derived from interlocking schedules (cf. Berryman \& Nevin, 1962). This interlocking procedure allowed us to generate the three sets of parallel MFFs, differing in (positive) slope. An interlocking schedule is defined by a procedure in which a fixedratio value is decreased linearly as a function of postfood time according to the function

$$
M(t)=K-J t, \quad 1 \leq M(t)
$$

where $M(t)$ is the effective ratio value $t \mathrm{~s}$ after food; $K$ is the initial ratio (e.g., 32 responses per pellet); $J$ is the rate of decre- 


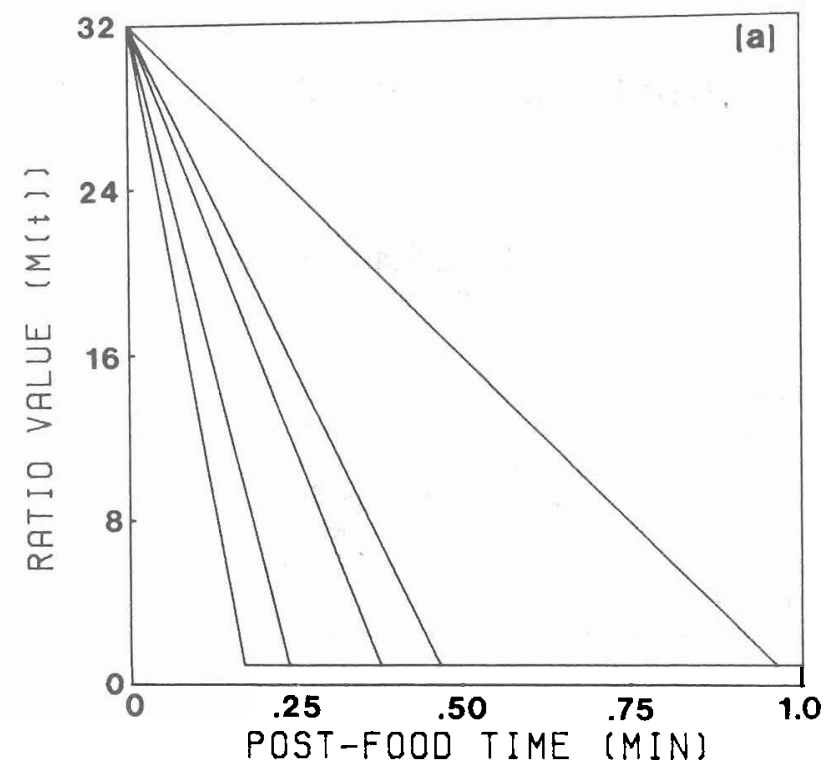

Figure 1. Decreasing ratio value as a function of five different rates of decrement for one set (IR32) of interlocking schedules.

ment (e.g., 3.03 response decrements/s); and $t$ is postfood time in seconds.

Figure 1 shows, for one set of interlocking schedules (IR32), how the initial ratio value (IR32) was decremented as a function of postfood time. The initial ratio value was decremented at the five different rates illustrated in the figure to a value of FR 1 (fixed ratio 1), because at least one response was always required for food delivery. Three complete sets of these interlocking schedules were created by selecting three different values for the initial ratio value: 64,32 , and 16 . The five rates of decrement were the same for each of the three sets of interlocking schedules. The three different sets of schedules are not depicted in Figure 1; however, they would be represented by lines converging on three different intercepts on the ordinate, representing the three different initial ratio values. The downward sloping lines can be described by Equation 1, where the slope is defined by $J$ (the rate of decrement) and the intercept by $K$ (the initial ratio value).

We can derive the MFF for Equation 1 by the realization that molar reinforcement and response rates are equivalent to the average postfood rates. By allowing $x$, the molar response rate, to equal $M(t) / t$ (the average local response rate during interfood intervals) and $R$, the molar food rate, to equal $1 / t$ (the average local food rate), we obtain a function that is independent of the obtained local response pattern:

$$
x=K R-J .
$$

Figure 2 shows how Equation 2 becomes a set of parallel, linear MFFs in molar response/food-rate space where the slope is determined by $K$ (the initial ratio value when postfood time $=0$ ), and the response rate intercept by $J$ (the rate of ratio decrement).

Equation 2 can be rearranged in terms of $R$ as

$$
R=x / K+J / K \text {. }
$$

Minimum-distance and utilitv-maximizing (and some other) optimality models predict that when MFF slope is held constant, but intercept varies, the locus of steady-state response rate/food-rate points (response function) will be linear, with a slope that depends critically on MFF slope. Models differ on the predicted effect of a change in MFF slope, but all molar optimality models predict some effect of MFF slope on response-function slope.

Because an understanding of these predictions is important for the interpretation of the experiments that follow, we derive these predictions for Staddon's (1979) minimum-distance model and Rachlin, Kagel, and Battalio's (1980) utility-maximizing model.

The predicted response function for a minimum-distance model can be derived by minimizing its cost function subject to the constraints of the schedule. For example, the cost function for Staddon's (1979) minimum-distance model (assuming that the free-operant level of barpressing is near zero) is

$$
C=a\left(R-R_{0}\right)^{w}+b X^{w},
$$

where $R$ is the molar reinforcement rate, $X$ is the molar response rate, and $a$ and $b$ are scaling constants representing the relative costs of deviations in each direction. The exponent $w$ is a constant that defines the characteristics of food-rate/response-rate space, usually assumed to be equal to 2 . The only active constraint is the molar feedback function (MFF) for the interlocking schedule, Equation 3.

A simple procedure for minimizing a function subject to constraint is to form the Lagrangian equation:

$$
L(R, X, \lambda)=a\left(R-R_{0}\right)^{w}+b X^{w}+\lambda(R-X / K-J / K),
$$

and taking partials with respect to all three variables:

$$
\begin{aligned}
& \partial L / \partial R=a w\left(R-R_{0}\right)^{w-1}+\lambda \\
& \partial L / \partial X=b w X^{w-1}-\lambda / K \\
& \partial L / \partial \lambda=R-X / K-J / K .
\end{aligned}
$$

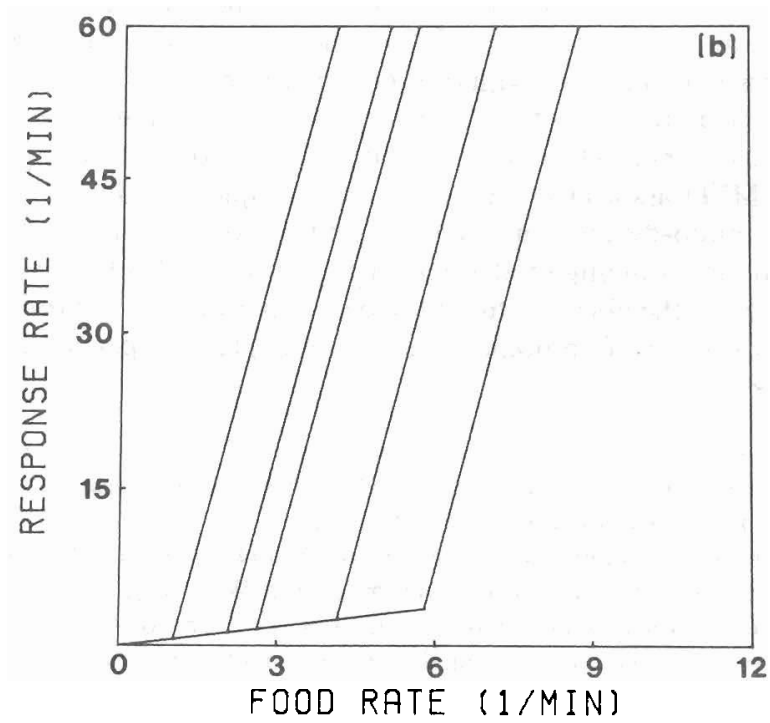

Figure 2. Molar feedback functions for the interlocking schedules nresented in Figure 1 


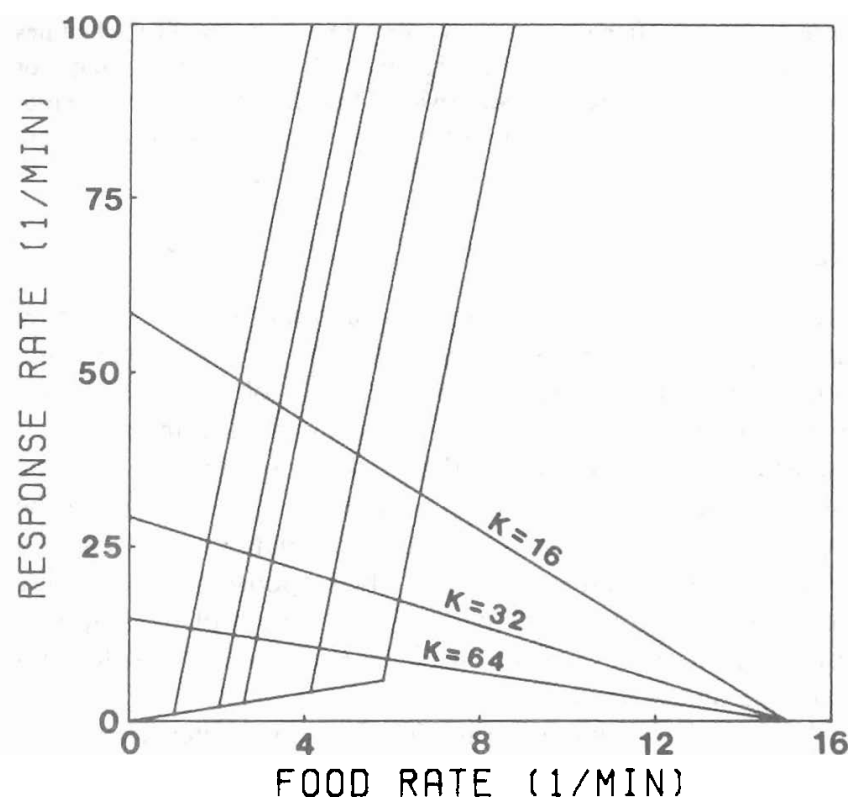

Figure 3. One set of molar feedback functions (IR32) and Staddon's (1979) minimum-distance predictions of the molar response functions as the initial ratio value, $K$, is varied.

Setting the partials equal to zero and combining Equations 6 and 7 gives

$$
a w\left(R-R_{0}\right)^{w-1}+\left(b w X^{w-1}\right) / K=0,
$$

and simplifying gives the predicted response function:

$$
X=(-a / b K)^{1 /(w-1)}\left(R-R_{0}\right) .
$$

Equation 9 predicts a linear relation between response rate and food rate with slope $(-a / b K)^{1 /(w-i)}$. Thus, the response function should be linear with negative slope with any beginning ratio value, $K$, and should be independent of the actual rate of decrease of the response requirement, $J$. The slope of the response function must vary as the beginning ratio value, $K$, is changed. Therefore, higher initial ratio values should produce flatter negatively sloped response functions than should lower initial ratio values. Figure 3 depicts one set of MFFs $(K=32)$ with Staddon's three predicted response functions as the initial ratio value, $K$, is varied.

The predicted response function for the Rachlin et al. (1980) utility-maximizing model can be derived by using the same technique. Their utility function is

$$
U=a R^{y}+b X^{y},
$$

in which $U$ represents the utility of the package of rewards, $R$, and responses, $X$. The constants $a$ and $b$ represent preference for $R$ and $X$, and $y$ is a constant exponent that represents the substitutability between the two activities. Equation 10 assumes that utility is calculated by summing the values of each of the individual activities that occur over the period of measurement. However, lever pressing is usually considered a costly activity, rather than being positively valued. Rachlin, Battalio, Kagel, and Green (1981) remind us that lever pressing is costly by inverting the response rate axis, as we have done in Figure 4.

The predicted response function can be derived analytically only after modifying Equation 10 to incorporate lever pressing as a costly activity. ${ }^{1}$ The modified utility function that allows increasingly valued indifference contours with lower response rates is

$$
U=a R^{y}+b(C-X)^{y},
$$

in which $C$ represents the response rate at the point where the two axes intersect (60 responses per minute in Figure 3).

As before, the predicted response function can be derived by forming the Lagrangian equation, with the MFF as the only active constraint:

$$
L(R, X, \lambda)=a R^{y}+b(C-X)^{y}+\lambda(R-X / K-J / K) .
$$

Solving the three resulting partial derivatives gives the predicted response function:

$$
X=C-(a / K b)^{1 /(y-1)} R .
$$

Equation 13 predicts a linear response function with negative slope, $-(a / K b)^{1 /(y-1)}$. Figure 4 depicts the predicted molar response functions as the initial ratio value, $K$, is varied. These depicted functions have negative slopes because the response rate axis is inverted. Increasing $K$, the slope of the MFF, produces steeper predicted response functions. Therefore, lower initial ratio values should produce flatter negatively sloped response functions than should higher initial ratio values, a prediction opposite to that of Staddon's minimum-distance model above.

It is clear that predictions from both molar optimality models depend critically on the slopes of the schedules' molar feedback functions.

\section{Method}

Subjects. Four female albino rats, approximately 4 months old at the beginning of the experiment, were used. By restricting food intake, the animals (IL-1, IL-2, IL-3, and IL-4) were maintained at approximately $80 \%$ of their preexperimental free-feeding weights throughout

\footnotetext{
'The incorporation of responding as a costly activity into the utility function is not as straightforward as it might seem. Equation 10 states that utility is the sum of the values of the individual constituent activities. However, although value and cost are inversely related, it is not correct simply to negate the sign of that portion of the equation representing the value of responding, $b X^{\nu}$. Rachlin, Battalio, Kagel, and Green (1981) demonstrate that responding is costly by replacing responding with leisure in their figures and by inverting the response axis to represent the amount of lever pressing. Predictions are made graphically rather than analytically, and the original utility function is never altered to represent the cost of responding. Our attempt to find analytic solutions was very useful to us because it helped identify the assumptions made by viewing responding as a costly activity. For example, the representation of responding and leisure as inversely related activities on the same axis in temporal units assumes that time taken from one of these activities must be allocated to the other, that is, the time taken $u p$ by both activities is constant $\left(\mathrm{T}_{X}+T_{L}=C\right)$. The absence of a contribution of time spent eating follows from the fact that they assume their utility function represents binary choice: In a situation with three activities (eating, lever pressing, and leisure), animals must choose (rather, they allocate time) between eating and the package of lever pressing and leisure before they choose how much time to spend lever pressing or in leisure. Thus, time spent lever pressing is taken from time spent in leisure and never from time spent eating. Therefore, Equation 11 above makes precisely the same assumptions as does Rachlin et al.'s (1981) inversion of the response axis.
} 


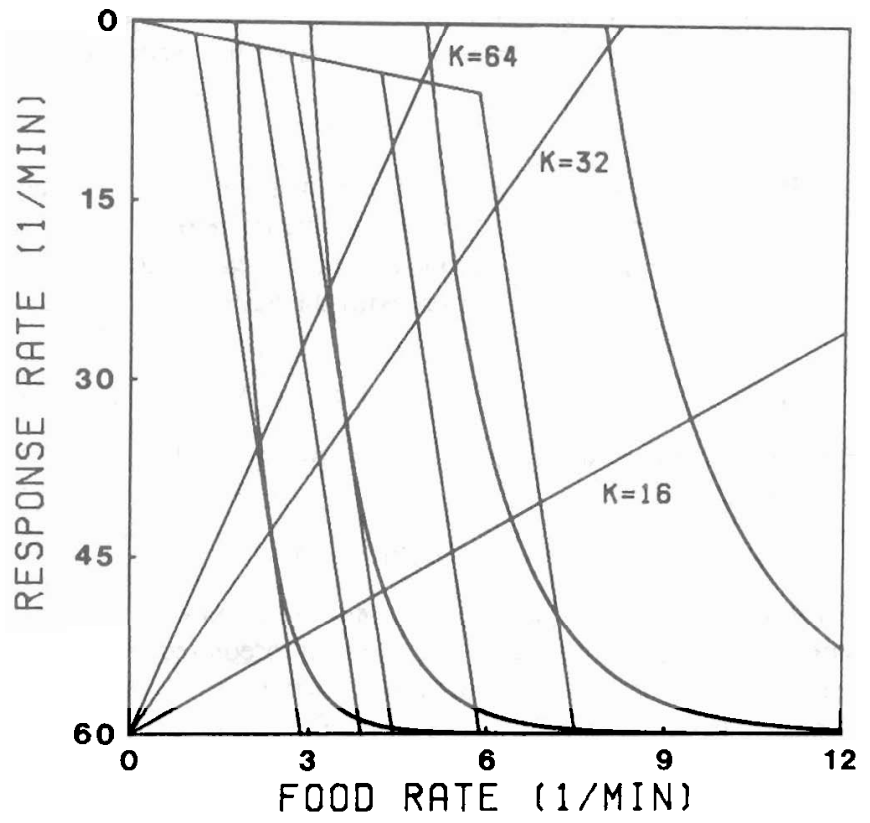

Figure 4. One set of molar feedback functions (IR32) and Rachlin et al.'s (1980) indifference contours and predicted molar response functions as the initial ratio value, $K$, is varied.

the experiment. Tap water was continuously available in the home cages. Body weights were maintained by supplemental feedings of Purina Rat Chow following each daily experimental session. Throughout the experiment all animals were individually housed under constant illumination.

Apparatus. Experimental sessions were conducted in a Plexiglas Skinner box measuring $23 \times 28 \times 15(\mathrm{~L} \times \mathrm{W} \times \mathrm{H}) \mathrm{cm}$ enclosed in a sound-attenuating enclosure. The response lever and the food cup were located on the front panel centered $4 \mathrm{~cm}$ above the floor and separated by $10 \mathrm{~cm}$. A force of approximately $45 \mathrm{~g}$ was required to operate the response lever. A Gerbrands (Model D-1) pellet dispenser delivered 45$\mathrm{mg}$ food pellets into the cup. A white lamp provided chamber illumination during the session only. White noise masked extraneous sounds. A microcomputer controlled all experimental manipulations and recorded all experimental events with $1 / 15^{-}$s resolution. Data were transfered to another computer for analysis.

Procedure. Each animal was first trained to lever press for food reinforcement by a successive approximation procedure. Following lever-press training, all of the animals were exposed to three sets of interlocking schedules with positive slopes. The first set of schedules was defined by a fixedratio (FR) 32, which was decremented at five different postfood rates. These different rates produced five parallel MFFs with positive slope. We term this series of parallel MFFs $I R 32$. The different rates $(J)$ at which each set of FR schedules was decremented were $3.03,2.13,1.37,1.08$, and 0.54 response decrements/s. The ratio value was never decremented below FR 1-food was never delivered freely. For example, on the FR 32 set of schedules, at the beginning of a session and immediately following each reinforcement (i.e., postfood time $=0$ ) the FR value was 32 .

The second and third sets of parallel MFFs were defined by fixedratios 16 and 64 (IR 16 and IR64) and used the same five decrement rates used with IR32. Thus, the animals were exposed to $3 \times 5=15$ different reinforcement schedules. These schedules correspond to three sets of five linear, positive MFFs. Each session was terminated after 100 pellets were delivered. Sessions were conducted daily on each schedule until response rates appeared stable; that is, there was neither an increasing nor a decreasing trend in response rates for the last 5 days. Response functions for each of the three sets of interlocking schedules were replicated after presenting the first 15 schedules. The schedules were presented for 5 days during the first replication and for 3 days for the schedules that were replicated twice. The order of schedule presentations and the number of sessions that each schedule was presented are given in Table 1.

\section{Results}

Figure 5 presents the three sets of response functions and their replications for each of the 4 animals. The response functions represent the average relation between obtained food rates and response rates for each animal across the last four sessions under each schedule during the first exposure and across the last three sessions during each replication. The slopes and the regression coefficients of these response functions are presented in Table 2. For each set of MFFs, the response functions were approximately linear. Furthermore, the slopes of the response functions were very similar, with the exception of that for animal IL-2 on the IR64 schedule.

A test of the general linear model allows for a determination of the probability that the slopes of the three response functions are different from one another. Table 2 contains the results from this analysis for each subject. The slopes of the response functions were not significantly different $(p>.05)$ for any of the subjects, even for IL-2, whose slopes varied over the largest range of any animal.

Figure 6 presents typical patterns of postfood responding from similar schedules $(J=1.37$ response decrements/s) from the set of interlocking MFFs for IR 16, IR32, and IR64. The figure shows the first six postfood responses averaged across a single session for 1 animal. The postreinforcement pause for each schedule is represented by the point intersecting each curve and the abscissa. The pause times did not vary for different values of $J$.

\section{Discussion}

It is useful to compare the obtained response functions with the predicted response functions from Staddon's (1979) model and Rachlin et al.'s (1980) model. Figure 7 contains the three

Table 1

Summary of Conditions for Experiment 1

\begin{tabular}{lcccc}
\hline IR & $\begin{array}{c}\text { Rate of FR } \\
\text { decrement }\end{array}$ & $\begin{array}{c}\text { No. of } \\
\text { sessions }\end{array}$ & $\begin{array}{c}\text { Sessions of } \\
\text { rep. 1 }\end{array}$ & $\begin{array}{c}\text { Sessions of } \\
\text { rep. 2 }\end{array}$ \\
\hline 32 & 0.54 & 25 & 5 & 3 \\
32 & 1.08 & 24 & 5 & 3 \\
32 & 1.37 & 14 & 5 & 3 \\
32 & 2.13 & 12 & 5 & 3 \\
32 & 3.03 & 11 & 5 & 3 \\
64 & 0.54 & 5 & 5 & \\
64 & 1.08 & 5 & 5 & \\
64 & 1.37 & 5 & 5 & \\
64 & 2.13 & 5 & 5 & \\
64 & 3.03 & 5 & 5 & \\
16 & 0.54 & 5 & 5 & \\
16 & 1.08 & 5 & 5 & \\
16 & 1.37 & 5 & 5 & \\
16 & 2.13 & 5 & 5 & \\
16 & 3.03 & 5 & 5 & \\
\hline
\end{tabular}

Note IR = initial ratio; FR = fixed ratio; No. = number; Rep. $=$ replication. 


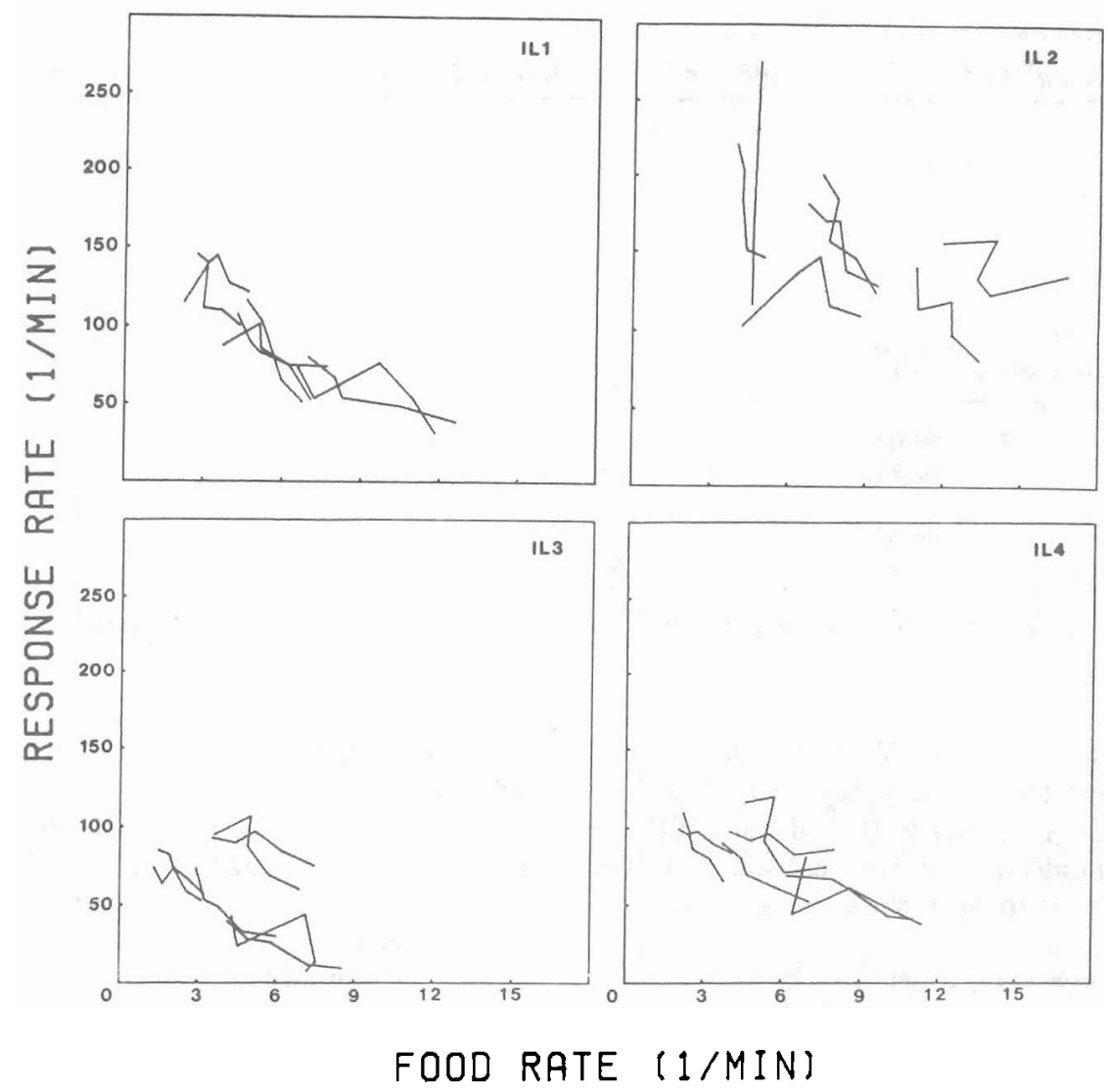

Figure 5. Obtained molar response functions and their replications from three schedule conditions for each subject (IL1-IL4) in Experiment 1.

sets of response functions averaged across replications for each animal. Also depicted are Staddon's three predicted response functions, which converge on the abscissa. The predicted response functions from the Rachlin et al. model converge on the ordinate at the point of best fit of the obtained response functions. This figure is useful because it depicts the precise slopes predicted for the values of $K$ that we used, because no other

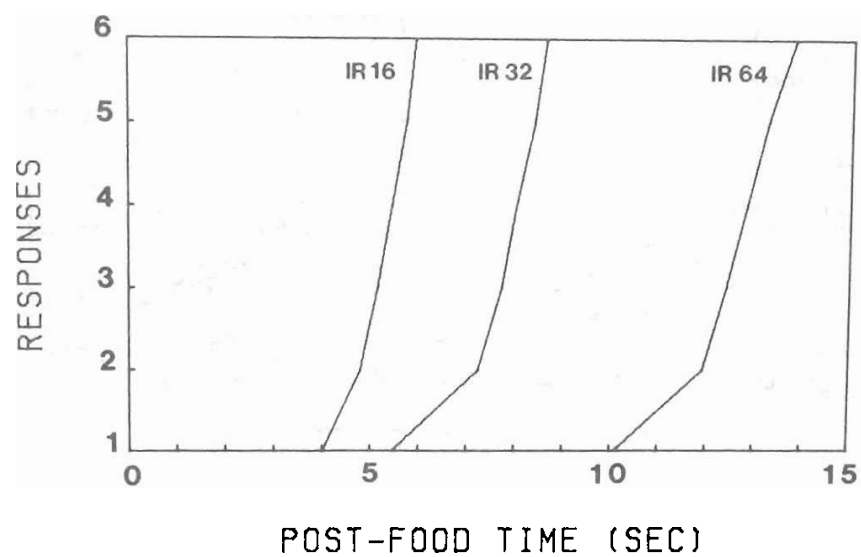

Figure 6. Averaged cumulative records for the first six postfood responses for a single session on the interlocking schedules with beginning ratio (IR) values of 16,32 , and 64 . parameters in either model can change from condition to condition. Therefore, the insignificant differences in the obtained response functions are not sufficient to allow the conclusion that these results support either model.

Independent of slope predictions, both models make the additional, and equally important, prediction of a change in the average response rate from condition to condition. For example, it is clear in Figure 7 that each obtained response function should be displaced vertically with respect to one another. That is, obtained data points should lie along the predicted functions, which vary widely along the ordinate. Although some variation in response rate was obtained (especially for IL-3), obtained response rates did not vary in accordance with either model.

Evidently all three sets of feedback functions in this experiment yielded similar linear response functions. We cannot rule out the possibility of a small, nonsignificant slope change. The theoretical question of interest is whether we should expect a larger change, given that molar optimality theories must account for response-function differences between interval and ratio schedules solely by means of the different MFFs associated with them. Our MFF-slope manipulation should have been sufficient to change response-function slope. Variable-interval (VI) schedules have a curved MFF whose slope varies from 1 at very low response rates to infinity at very high rates. Ratio schedules, over the usual range, have slopes varying from $I$ to perhaps 100; the MFFs in this experiments had slopes ranging from 16 to 64 . Molar optimality theories attribute the generally 
Table 2

Comparison of the Slopes and the Correlation Coefficients for the Interlocking Response Functions (IR) in Figure 4

\begin{tabular}{|c|c|c|c|c|}
\hline $\begin{array}{c}\text { Condition \& } \\
\text { animal }\end{array}$ & IR 16 & IR 32 & IR 64 & Test of general linear model \\
\hline $\mathrm{IL}-1$ & & & & $F(2,9)=2.056, p>.15$ \\
\hline Slope & -8.78 & -15.76 & -11.85 & \\
\hline Correlation & -.95 & -.98 & -.83 & \\
\hline IL-2 & & & & $F(2,9)=2.811, p>.10$ \\
\hline Slope & -10.79 & -14.50 & -59.81 & \\
\hline Correlation & -.84 & -.89 & -.82 & \\
\hline IL-3 & & & & $F(2,9)=1.679, p>.20$ \\
\hline Slope & -7.08 & -12.02 & -13.89 & \\
\hline Correlation & -.81 & -.95 & -.98 & \\
\hline IL-4 & & & & $F(2,9)=1.400, p>.25$ \\
\hline Slope & -6.52 & -4.86 & -12.99 & \\
\hline Correlation & -.90 & -.90 & -.99 & \\
\hline
\end{tabular}

Note. The general linear model determines the probability that the slopes of the obtained response functions are different from each other.

positively sloped response functions on VI to the positive acceleration of the MFFs, and the generally negatively sloped response functions on ratio schedules to their linear MFFs. In other words, substantial differences in response-function slope are attributed to differences in MFF slope in the same range that we studied here.

The local patterns of responding engendered by the schedules were typical of interlocking and fixed-ratio schedules (cf. Figure 6). The postreinforcement pause increased as the value of the ratio increased. This regularity at the molecular level demonstrates that the animals were sensitive to local properties of the different interlocking schedules. Therefore, it is clear that the absence of variation in response-function slope could not be due to a failure to discriminate between conditions.

\section{Experiment 2}

In Experiment 1, as the slope of each set of positively sloped MFFs was varied, the slope of the corresponding response function remained nearly constant. In Experiment 2 we looked at sets of MFFs with negative slopes to see if (a) the response functions were similarly insensitive to MFF slope changes and (b) if the response functions were different from those found in Experiment 1. The schedule studied in Experiment 2 was similar to the negatively sloped linear VI schedule used by Vaughan and Miller (1984). This schedule is a variable interval in which a reinforcement counter is incremented when each interval is completed and decremented after the completion of a fixed number of responses. Thus, higher response rates yield lower reinforcement rates (unless response rate is very low) as with DRL (differential-reinforcement-oflow-rate) schedules.

The molar feedback function for the negatively sloped portion of these schedules is

$$
R=V-x / P,
$$

where $R$ is the overall food rate, $x$ is response rate, $V$ is the maximum programmed food rate for the stored-reinforcer variable-interval schedule (approximately a constant), and $P$ is the ratio value. As $x \rightarrow 0, R \rightarrow x$, because a response is always required for each food delivery, the MFF is a triangle as shown in Figure 8 . These schedules yield negatively sloped MFFs that are nearly symmetrical to the positive MFFs for the interlocking schedules used in Experiment 1.

The predicted molar response functions from Staddon's (1979) minimum-distance model can be derived by forming the Lagrangian equation from his cost function and the two active constraints: the one formed by the MFF in Equation 14, and $R \leq X$, the minimum requirement for food delivery:

$$
\begin{aligned}
& L\left(R, X, \lambda_{1}, \lambda_{2}\right) \\
& \quad=a\left(R-R_{0}\right)^{2}+b X^{2}+\lambda_{1}(V-X / P-R)+\lambda_{2}(X-R) .
\end{aligned}
$$

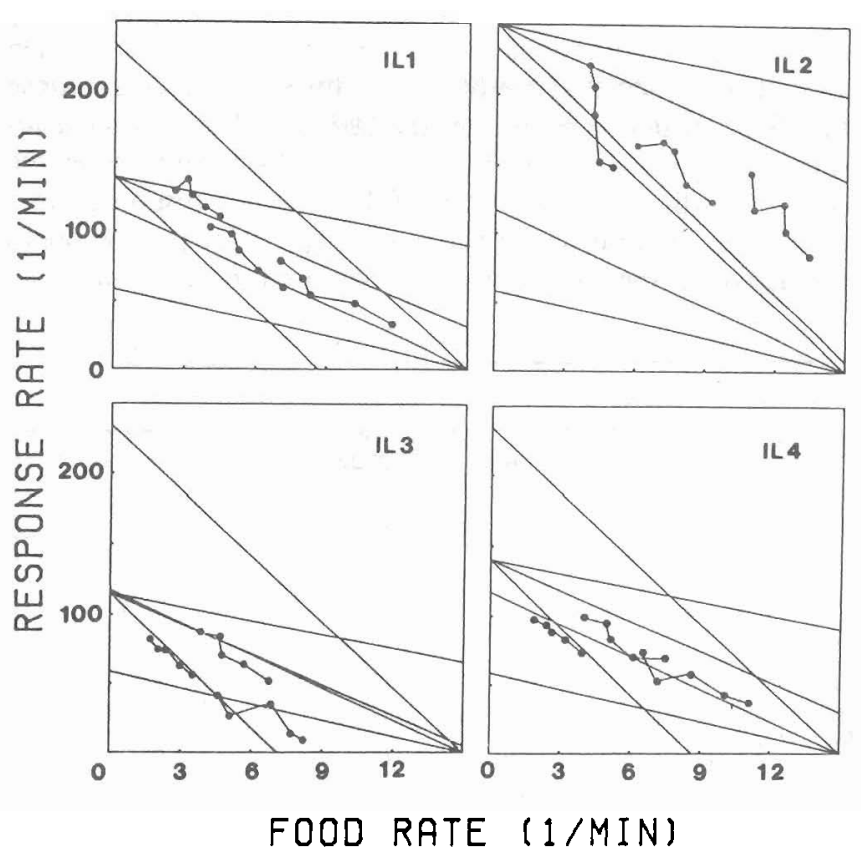

Figure 7. The lines with individual data points represent the molar response functions averaged across replications in Experiment 1 for subjects IL1-IL4. (Lines converging on the abscissa represent the three predicted molar response functions from Staddon's, 1979, optimality model. Lines converging on the ordinate represent the three predicted molar response functions from Rachlin et al.'s, 1980, optimality model.) 


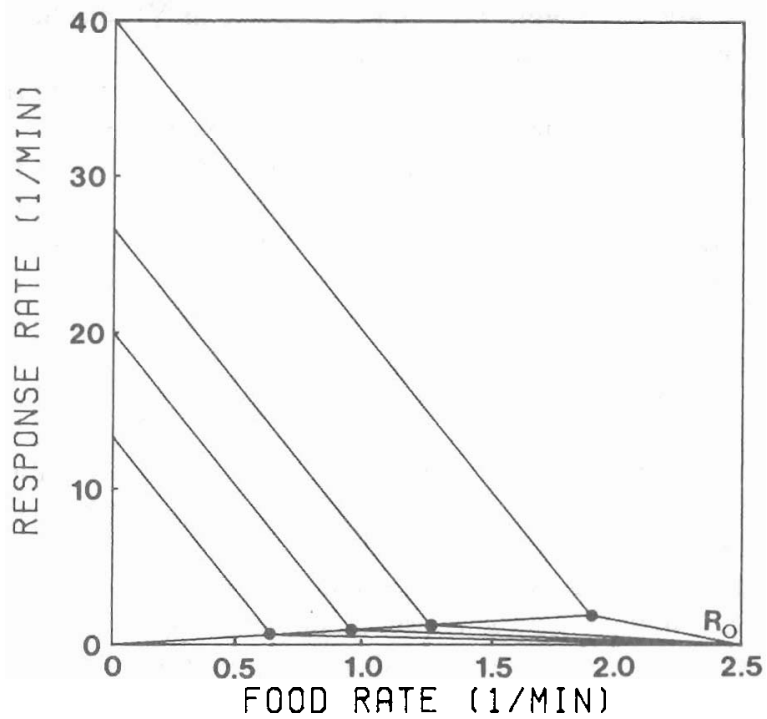

Figure 8. One set of molar feedback functions (SR20) and Staddon's (1979) predictions of the points on the molar feedback functions representing the minimum distance to the regulated point, $R_{0}$. (The predicted molar response function connects these points.)

Taking all four partials, eliminating $\lambda_{1}$ and $\lambda_{2}$, and substituting yields the predicted molar response function:

$$
X=R=V P /(P+1) .
$$

The predicted response function of Equation 16 is the set of points connecting the two constraints, that is, where the FR-1 line, $X=R$, intersects the negatively sloped MFFs. This point of intersection is determined exclusively by the programmed food rate, $V$, and the ratio value, $P$. Figure 8 shows the negatively sloped MFFs with the points representing the minimum distance to $R_{0}$ predicted by Staddon's model.

The same technique can be used to derive the predicted response function for the Rachlin et al. (1980) utility-maximizing model. Both constraints are again active, and the Lagrangian equation is

$$
\begin{aligned}
& L\left(R, X, \lambda_{1}, \lambda_{2}\right) \\
& \quad=a R^{y}+b(C-X)^{y}+\lambda_{1}(V-X / P-R)+\lambda_{2}(X-R) .
\end{aligned}
$$

Taking all four partials, eliminating $\lambda_{1}$ and $\lambda_{2}$, and substituting yields the predicted molar response function:

$$
X=R=V P /(P+1),
$$

which is exactly the same as Equation 16, the prediction from Staddon's minimum-distance model. Thus, the point on each MFF which crosses the highest indifference contour is the rightmost tip of each triangle. Figure 9 shows the Rachlin et al. predictions with the MFFs from the linear VI (with FR subtract) procedure. Thus, unlike Experiment 1, where Rachlin et al. and Staddon make opposite predictions, they make identical predictions for this experiment.

\section{Method}

Subjects. Four female albino rats (LV-1, LV-2, LV-3, and LV-4), approximately 10 months old at the beginning of the experiment, were used. All animals had some previous experience with fixed-ratio and variable-interval schedules. The animals were maintained at approximately $85 \%$ of their preexperimental free-feeding weights throughout all phases of the experiment by restricting food intake. Tap water was continuously available in the home cages. Body weights were maintained by supplemental feedings of Purina Rat Chow following each daily experimental session. Throughout the experiment all animals were individually housed under constant illumination.

Apparatus. Experimental sessions were conducted in a Plexiglas Skinner box measuring $30.5 \times 23 \times 15(\mathrm{~L} \times \mathrm{W} \times \mathrm{H}) \mathrm{cm}$ enclosed in a sound-attenuating box. The response lever and the food cup were located on the front panel centered $5 \mathrm{~cm}$ above the floor and separated by $6 \mathrm{~cm}$. A force of approximately $45 \mathrm{~g}$ was required to operate the response lever. A Gerbrands (Model D-1) pellet dispenser delivered 45-mg food pellets into the cup. A white lamp provided chamber illumination during the session only. White noise masked extraneous sounds. A microcomputer controlled all experimental manipulations and recorded all experimental events with $1 / 15$-s resolution. Data were transferred to another computer for analysis.

Procedure. Each animal was exposed to two sets of linear variableinterval (LVI) schedules with negative slopes. The first set of schedules was generated by subtracting an FR 20 schedule from four different linear variable-interval schedules (we term this series SR20), and the second set was generated by subtracting an FR 15 (SR 15). Thus, the animals were exposed to $2 \times 4=8$ reinforcement schedules. The LVI schedules used were LVI 30, LVI 45, LVI 60, and LVI 90 s. These schedules consisted of 16 intervals derived by the procedure described by Fleshler and Hoffman (1962). When an interval elapsed, the reinforcement store was incremented by one, and the timer continued. When the FR 20 was satisfied, the store was decremented by 1 . Hence, the store could be driven to negative values by high response rates. A response that occurred when the value of the store was positive resulted in the delivery of one food pellet (Noyes $45 \mathrm{mg}$ ) and decremented the store value by 1 . The order of schedule presentations was mixed, and each schedule was presented until response rates appeared stable; that is, there was neither an increasing nor a decreasing trend in response rates over the last 5 days. Sessions were conducted daily and were terminated

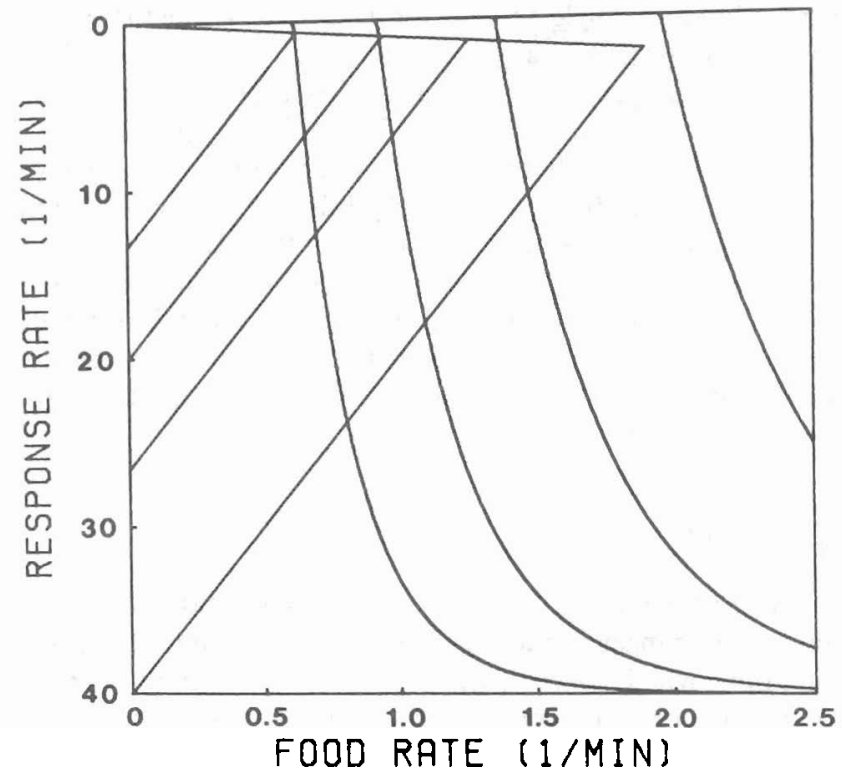

Figure 9. One set of molar feedback functions (MFFs, SR20) and Rachlin et al.'s (1980) indifference contours. (The predicted response function connects the points where the highest indifference contours intersect each MFF.) 
Table 3

Summary of Conditions for Experiment 2: Order of Schedule Presentations

\begin{tabular}{ccc}
\hline Linear VI value (in s) & FR value & No. of sessions \\
\hline 60 & 20 & 16 \\
90 & 20 & 13 \\
30 & 20 & 16 \\
45 & 20 & 10 \\
30 & 15 & 11 \\
60 & 15 & 10 \\
45 & 15 & 12 \\
90 & 15 & 12 \\
\hline
\end{tabular}

Note. $\mathrm{VI}=$ variable interval; $\mathrm{FR}=$ fixed ratio

after $60 \mathrm{~min}$. The order of conditions and the number of sessions for each condition are presented in Table 3.

\section{Results}

The results for all 4 animals are presented in Figure 10. The lines with negative slope represent one of the two sets of MFFs. The lines through the symbols represent the corresponding response functions for each animal averaged over the last 5 days that each schedule was in effect. Table 4 presents the slopes and the regression coefficients for the response functions in Figure 10. In each case, the points are well represented by straight lines through the origin. The response functions were all approximately linear. Table 4 also presents the results of a test of the general linear model, which demonstrates that the slopes of the two response functions did not differ significantly $(p>.05)$ as a function of the two MFF slopes for any animal.

Figure 11 presents typical patterns of postfood responding on an LVI 30-s and an LVI 90-s schedule with FR-20 subtract requirements. The figure shows the first 20 postfood responses averaged over a single session for 1 animal. Behavior on this schedule is not temporally organized with respect to postfood time: The averaged cumulative record is approximately linear, with no postreinforcement pause. The steeper slope for the LVI 30 -s schedule shows that the animals were sensitive to the local properties of the different LVI schedules. We cannot demonstrate sensitivity to the different slopes of the MFFs at the local level because if there is no postfood pause (as Figure 11 demonstrates), the pause duration cannot be a function of the schedule in effect. Other measures of sensitivity such as running response rate would probably have to show up as a molar rate difference, which was not observed.

\section{Discussion}

As in Experiment 1, variation in MFF slope did not produce significant changes in the slopes of the corresponding response functions, which were linear, as before-but with positive rather than negative slope. This invariance in response-function slope did not reflect insensitivity to the procedure: All animals showed consistent changes in response rate under different schedule parameter values (i.e., the VI values). In this respect our rats differed from the pigeons used in the experiment of Vaughan and Miller (1984), which showed no consistent rela- tion between response rates and the particular VI schedule in effect as the FR subtract was manipulated.

It is again instructive to compare the obtained response functions depicted in Figure 10 with the points predicted by both Staddon's (1979) minimum-distance model illustrated in Figure 8 and Rachlin et al.'s (1980) value-maximizing model depicted in Figure 9. Both models predict that responding should lie on the FR-1 constraint. However, the obtained response rate was substantially higher than either model predicts.

\section{General Discussion}

In Experiment 1 the positively sloped MFFs produced linear, negatively sloped response functions similar to those produced by ratio schedules (Ettinger \& Staddon, 1983). In Experiment 2, negatively sloped MFFs produced linear, positively sloped response functions. In each case, as the slope of the MFF was changed, the slope of the corresponding response function did not change systematically. These results pose serious problems for models of schedule performance that assume direct sensitivity to the MFF (e.g., the molar optimality models of Kagel et al., 1980; Rachlin \& Burkhard, 1978; Staddon, 1979; Timberlake, 1980).

Molar optimality models have been most successful in explaining the difference between performance on ratio and interval schedules. Performance on these schedules differs in two main respects: (a) Over most of the usual range of values, VI performance follows a response function of positive slope, whereas the response function for FR is negatively sloped, or regulatory (e.g., Staddon, 1979); (b) when obtained reward rate is the same, response rate on the ratio schedule is generally higher than on the interval schedule (e.g., Lea \& Tarpy, 1982). Molar optimality theories can account for both differences, yet the same theory also requires slope changes that did not occur in this experiment. What alternatives exist?

Our results suggest a simple possibility. Typically, ratio procedures yield linear, negatively sloped, response functions over much of their range (e.g., FR 2 to FR 64), whereas interval procedures yield positively sloped response functions that are approximately linear over most of their range (e.g., VI values from $120 \mathrm{~s}$ to $30 \mathrm{~s}$ ). Rats and pigeons in typical operant-conditioning situations usually respond in such a way that these ranges barely overlap in terms of obtained reward rates. It is possible, therefore, that positively sloped response functions are the usual pattern at low (VI-typical) reward rates, whereas negatively sloped (regulatory) response functions are the usual pattern at higher (FR-typical) reward rates. Our data are consistent with this hypothesis, because the positively sloped MFFs used in Experiment 1 allowed the animals to obtain food at a relatively high rate, whereas the negatively sloped MFFs in Experiment 2 permitted only low rates that barely overlapped the rates in Experiment 1.

There are some other data that support this idea. For example, Allison (1980) has reported negatively sloped linear response functions on very short (7-14 s) variable-interval schedules of water reinforcement. The result over the more usual range of minutes is, of course, a positively sloped response function (cf. Herrnstein, 1970).

The existence of two modes, regulatory (negatively sloped response function) and nonregulatory (positively sloped response function) makes some sense in both functional and psychophys- 


\section{MOLAR FEEDBACK FUNCTIONS}

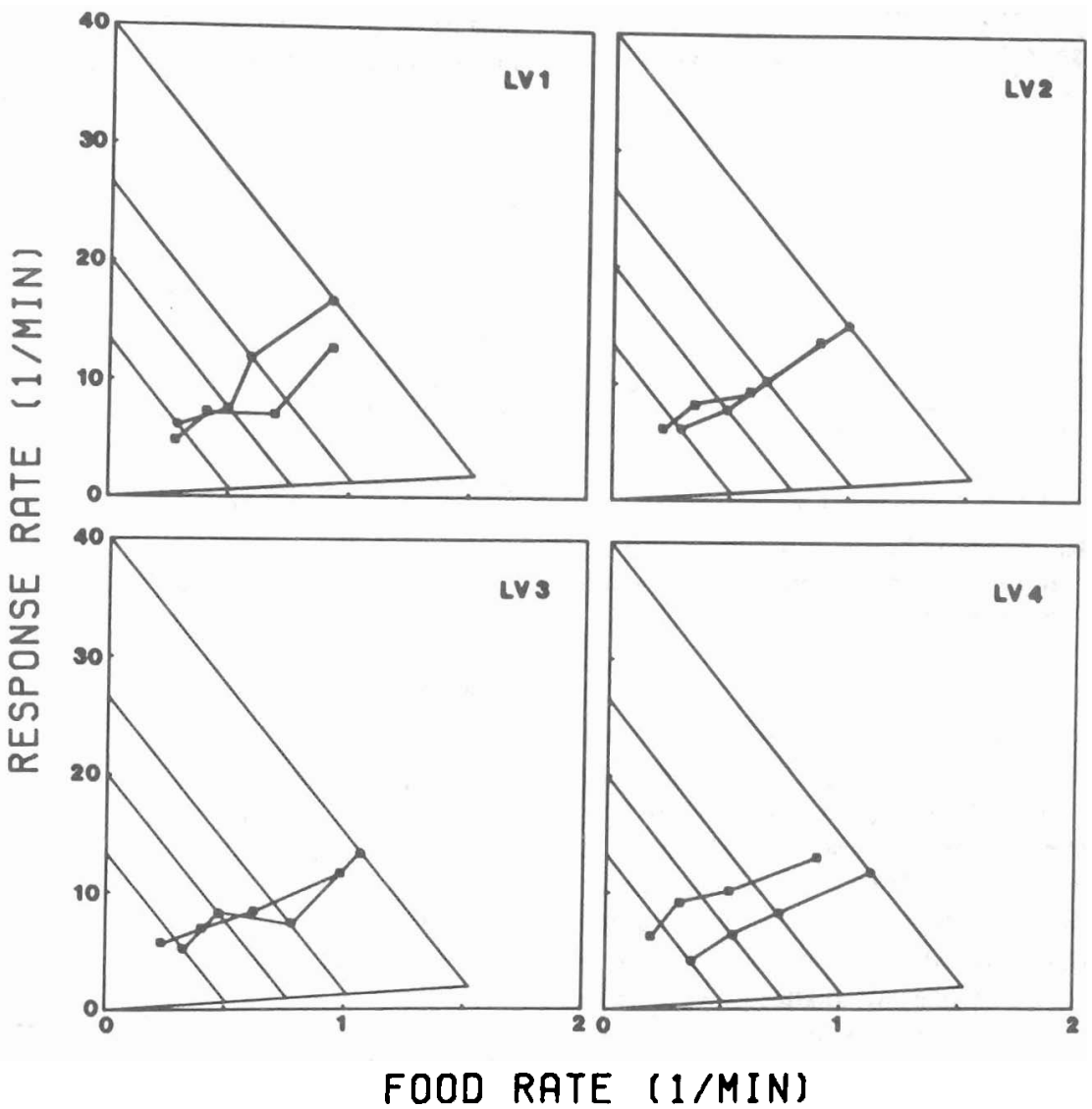

Figure 10. One set of molar feedback functions (solid lines) with corresponding obtained response functions (lines and symbols) for the two sets of linear variable interval schedules in Experiment 2. (LV1, LV2, LV3, LV4 = Animals 1, 2, 3, and 4.)

ical terms. From a functional point of view, food rate should be regulated only if the marginal cost of increased responding necessary is equal to the marginal benefit of the increased food obtained. Hence, regulation may not be worthwhile at very low food rates, where simple optimality dictates no responding at all. Nevertheless, if the animal completely ceases to respond, it cannot detect when things change for the better, so that considerations related to sampling dictate some responding even at very low payoff probabilities. The popular regulatory models (Kagel et al., 1980; Rachlin \& Burkhard, 1978; Staddon, 1979;

Table 4

Comparison of the Slopes and the Correlation Coefficients of the Response Functions in Figure 9

\begin{tabular}{lccc}
\hline $\begin{array}{c}\text { Condition \& } \\
\text { animal }\end{array}$ & Phase 1 & Phase 2 & Test of general linear model \\
\hline LVI-1 & & & $F(1,4)=0.109, p>.75$ \\
Slope & 8.94 & 8.72 & \\
Correlation & 0.94 & 0.96 & $F(1,4)=0.510, p>.50$ \\
LVI-2 & 8.39 & 10.40 & \\
Slope & 0.99 & 0.91 & $F(1,4)=1.911, p>.20$ \\
Correlation & 14.39 & 9.86 & \\
LVI-3 & 0.98 & 0.95 & $F(1,4)=1.442, p>.25$ \\
Slope & 15.07 & 16.36 & 0.94 \\
Correlation & 0.92 &
\end{tabular}

Note. Phase 1 consisted of the set of linear variable-interval (LVI) schedules with the FR 20 subtract, and Phase 2 consisted of the set of LVI schedules with the FR 15 subtract. The general linear model determines the probability that the slopes of the obtained response functions are different from each other. 


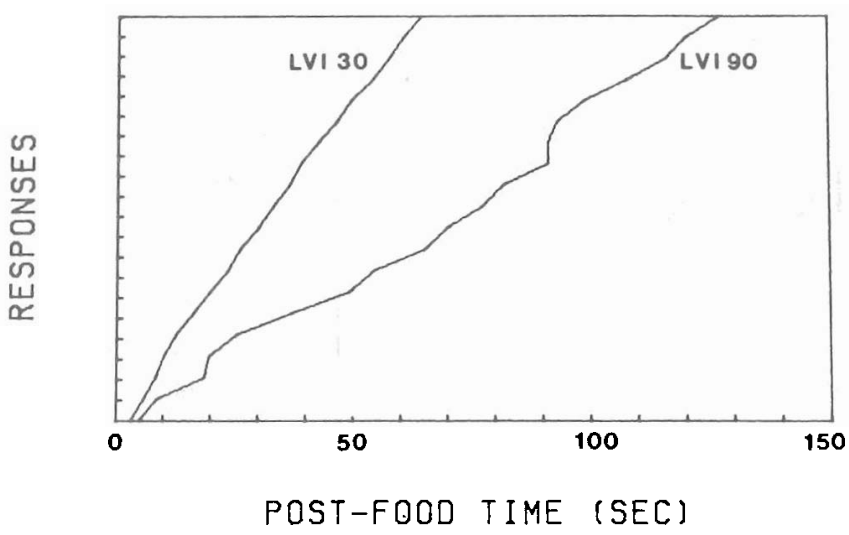

Figure 11. Averaged cumulative records for the first 20 postfood responses for a single session on a linear variable interval (LVI) and a linear VI 90-s schedule with FR 20 subtract.

Timberlake, 1980) all take account of this effect of payoff rate in the sense that all predict inverted-U-shaped response functions: a negatively sloped (regulatory) region at high reinforcement rates, a positively sloped region at low. Nevertheless, the extreme insensitivity to changes in MFF slope evident in our data and the linearity of both the regulatory and nonregulatory response functions are not consistent with published models.

From a psychophysical point of view, we might expect there to be some threshold for detecting differences in obtained reward rates: If the animal cannot detect small changes in reward rate, then it cannot adjust its responding accordingly - as required for regulation. At low reward rates the changes will obviously be smaller, and perhaps undetectable, precluding regulation.

Neither our data nor results in the literature compel the hypothesis that the different response functions in our two experiments reflect solely differences in obtained food rates. Another factor that might be responsible is the differential involvement of temporal factors, which were clearly involved in the interlocking schedules in Experiment 1 but not in the VI-FR-subtract schedules in Experiment 2, although this factor cannot account for the range of data in the literature. Nevertheless, additional experiments, comparing identical procedures (similar to those in both experiments) over different ranges of obtained food rates, will be necessary for a decisive test.

In these experiments the molar feedback function was not a sufficient description of the properties of the reward schedule responsible for the maintenance of steady-state behavior. In retrospect, is seems reasonable to ask why animals should be directly sensitive to properties of the MFF such as slope. This question implies that the feedback mechanisms responsible for maintaining steady-state behavior and those responsible for learning the schedule contingencies are different. Molar feedback functions were originally proposed to describe the feedback between the organism and the environment during both acquisition and steady-state performance (Baum, 1973). However, molar rates appeared to be the most appropriate measure for the feedback rela- tion for maintaining behavior on schedules, while few, if any, believed that animals depend on these molar rates (over entire sessions) during acquisition. We see no reason to suppose that the sources of feedback in the two situations or the underlying feedback mechanisms responsible for behavioral maintenance and learning are as different as current optimality theories assume.

\section{References}

Allison, J. (1980). Conservation, matching, and the variable-interval schedule. Animal Learning \& Behavior, 8, 185-192.

Baum, W. M. (1973). The correlational based law of effect, Journal of the Experimental Analysis of Behavior, 20, 137-153.

Berryman, R., \& Nevin, J. A. (1962). Interlocking schedules of reinforcement. Journal of the Experimental Analysis of Behavior, 5, 213223.

Ettinger, R. H., \& Staddon, J. E. R. (1983). The operant regulation of feeding: A static analysis. Behavioral Neuroscience, 97, 639-653.

Fleshler, M., \& Hoffman, H. S. (1962). A progression for generating variable interval schedules. Journal of the Experimental Analysis of Behavior, 5, 529-530.

Hermstein, R. J. (1970), On the law of effect. Journal of the Experimental Analysis of Behavior, 13, 243-266.

Hinson, J. M., \& Staddon, J. E. R. (1983). Matching, maximizing and hill climbing. Journal of the Experimental Analysis of Behavior, 40, $321-331$.

Kagel, J. H., Battalio, R. C., Green, L., \& Rachlin, H. (1980). Consumer demand theory applied to choice behavior of rats. In J. E. R. Staddon (Ed.), Limits to action: The allocation of individual behavior (pp. 237-267). New York: Academic Press.

Lea, S. E. G., \& Tarpy, R. M. (1982). Different demand curves from rats working under ratio and interval schedules. Behavioural Analysis Letters, 2, 113-121.

Prelec, D. (1982). Matching, maximizing and the hyperbolic reinforcement feedback function. Psychological Review, 89, 189-230.

Rachlin, H., Battalio, R., Kagel, J., \& Green, L. (1981). Maximization theory in behavioral psychology. The Behavioral and Brain Sciences, 4, 371-417.

Rachlin, H., \& Burkhard, B. (1978). The temporal triangle: Response substitution in instrumental conditioning. Psychological Review, 85 , $22-48$.

Rachlin, H., Kagel, J., \& Battalio, R. (1980). Substitutability in time allocation. Psychological Review, 87, 355-374.

Staddon, J. E. R. (1979). Operant behavior as adaptation to constraint. Journal of Experimental Psychology: General, 108, 48-67.

Staddon, J. E. R. (1982). Behavioral competition, contrast, and matching. In M. L. Commons \& R. J. Herrnstein (Eds.), Quantitative analyses of operant behavior: Matching and maximizing accounts (Vol. 2, pp. 243-261). Cambridge, MA: Ballinger.

Timberlake, W. (1980). A molar equilbrium theory of learned performance. In G. H. Bower (Ed.), The psychology of learning and motivation (Vol. 14, pp. 1-57). New York: Academic Press.

Vaughan, W. Jr., \& Miller, H. L. (1984). Optimization or responsestrength accounts of behavior. Journal of the Experimental Analysis of Behavior, 42, 337-348.
Received July 29, 1986

Revision received March 2, 1987 Accepted March 16, 1987 